\title{
Impactos psicoemocionais na hospitalização pediátrica: Percepções dos acompanhantes e a atuação da equipe de enfermagem
}

\author{
Psychoemotional impacts on pediatric hospitalization: Perceptions of companions and the \\ performance of the nursing team
}

Impactos psicoemocionales en la hospitalización pediátrica: Percepciones de acompañantes y actuación del equipo de enfermería

\section{Resumo}

O estudo tem por objetivo analisar as percepções e o impacto psicoemocional nos acompanhantes frente a hospitalização pediátrica, e a atuação da enfermagem nesse processo. Como método, foi utilizado uma revisão sistemática dos trabalhos produzidos entre os anos de 2016 a 2021 nas bases de dados: BDENF - Enfermagem, MEDLINE, LILACS, CINAHL e Index Psicologia; para isso foi seguida as diretrizes do PRISMA, utilizando os descritores "criança, percepção, família ou pais e enfermagem". Os critérios de inclusão se basearam em selecionar os artigos que se relacionavam com o objetivo de interesse, e fossem encontrados nas linguagens em inglês, português ou espanhol, e os de exclusão foram delineados em textos que não eram artigos. Diante dos resultados, ficaram evidentes a influência da hospitalização no psicossocial do acompanhante e do paciente pediátrico, e o quanto a equipe de enfermagem é fundamental nesse processo, desenvolvendo e aprimorando um cuidado humanizado que pode propiciar a diminuição da ansiedade tanto do paciente pediátrico quanto de seu acompanhante, contribuindo para a elevação da qualidade da assistência prestada, da confiança e do vínculo, consequentemente melhorando o processo de recuperação e reduzindo as chances de complicações.

Palavras-chave: Enfermagem; Hospitalização; Criança. 


\begin{abstract}
The study aims to analyze the perceptions and the psychoemotional impact on the companions in the face of pediatric hospitalization, and the role of nursing in this process. As a method, a systematic review of the works produced between the years 2016 to 2021 was used in the following databases: BDENF - Enfermagem, MEDLINE, LILACS, CINAHL and Index Psicologia; for this, the PRISMA guidelines were followed, using the descriptors "child, perception, family or parents and nursing". Inclusion criteria were based on selecting articles that were related to the objective of interest, and were found in English, Portuguese or Spanish languages, and exclusion criteria were outlined in texts that were not articles. In view of the results, the influence of hospitalization on the psychosocial of the companion and the pediatric patient became evident, and how fundamental the nursing team is in this process, developing and improving a humanized care that can provide the reduction of anxiety of both the pediatric patient and the patient. their companion, contributing to raising the quality of care provided, trusting and bonding, consequently improving the recovery process and reducing the chances of complications.
\end{abstract}

Keywords: Nursing; Hospitalization; Child.

\title{
Resumen
}

El estudio tiene como objetivo analizar las percepciones y el impacto psicoemocional sobre los acompañantes frente a la hospitalización pediátrica, y el papel de la enfermería en ese proceso. Se utilizó como método una revisión sistemática de los trabajos producidos entre los años 2016 a 2021 en las siguientes bases de datos: BDENF - Enfermagem, MEDLINE, LILACS, CINAHL e Index Psicologia; para ello se siguió la guía PRISMA, utilizando los descriptores "niño, percepción, familia o padres y enfermería". Los criterios de inclusión se basaron en la selección de artículos que estuvieran relacionados con el objetivo de interés y se encontraran en los idiomas inglés, portugués o español, y los criterios de exclusión se detallaron en textos que no fueran artículos. Ante los resultados, se hizo evidente la influencia de la hospitalización en la psicosocial del acompañante y del paciente pediátrico, y cuán fundamental es el equipo de enfermería en este proceso, desarrollando y perfeccionando un cuidado humanizado que pueda propiciar la reducción de la ansiedad tanto del paciente pediátrico y el paciente su acompañante, contribuyendo a elevar la calidad de la atención brindada, confiando y vinculando, consecuentemente mejorando el proceso de recuperación y reduciendo las posibilidades de complicaciones.

Palabras clave: Enfermería; Hospitalización; Niño.

\section{Introdução}

A internação em um processo de hospitalização pediátrica é um período potencialmente traumático tanto para a criança quanto para o acompanhante, pois há a quebra da rotina como: privações das práticas habituais, tanto familiar quanto escolar, somado ao contato intenso com um ambiente diferente, pessoas desconhecidas e principalmente pela realização de procedimentos que podem causar dor e desconforto (Sampaio et al., 2021). Então, é notório o impacto que causa na vida da criança e da sua família, pois o processo é envolto de muita tensão e ansiedade (Franzoi \& Martins, 2016). Com isso, estratégias que amenizam os sentimentos negativos sobre a hospitalização devem se tornar presentes, como o lúdico, que proporciona uma experiência a qual desenvolve habilidades e conhecimentos nas crianças (Rockembach, 2017).

Neste contexto, a ansiedade é um problema que pode manifestar-se de várias formas nas crianças, dependendo da faixa etária e do grau de desenvolvimento. Altos níveis de ansiedade podem levar ao desenvolvimento de alterações, tais como: aumento da frequência cardíaca, respiratória, da pressão arterial, bem como o aumento das chances de complicação na internação, no contexto comportamental (delírios, problemas na alimentação, irritabilidade, entre outros) e fisiológico, como dificuldade na recuperação (Binkowski et al., 2018; Sampaio et al., 2021).

O medo do desconhecido também é um potencializador da ansiedade no período de hospitalização, em ambos os sujeitos participantes desse momento, uma vez que irão vivenciar tais momentos juntas. Com isso, o acompanhante nem sempre será uma fonte plena de conforto e segurança para a criança, pois ele também terá suas preocupações e anseios. Assim, é importante que o enfermeiro reconheça as necessidades emocionais e seja capaz de prestar uma assistência que supra a necessidade de forma humanizada à criança e ao acompanhante, a fim de minimizar as dúvidas e preocupações e tornar esse momento o menos perturbador possível. (Sampaio et al., 2021).

As orientações que são realizadas pelo enfermeiro, podem favorecer em minimizar a ansiedade dos pacientes e dos seus acompanhantes antes, realizando uma ação educativa. O olhar do profissional de saúde no momento da hospitalização esclarece 
dúvidas e proporciona benefícios para a diminuição do medo e da ansiedade, juntamente com a recuperação deste paciente (Lucena et al., 2020).

A carência de orientações durante o período de internação pode resultar em dificuldades para realização do autocuidado e favorecer complicações, reinternações, aumento da dor, maior tempo de hospitalização, entre outros. Os enfermeiros são os profissionais que estão juntos do paciente por mais tempo e tal papel demanda mais atenção à educação em saúde, transmitindo informações acerca do assunto. Devido a isso, torna-se crucial a educação continuada desse profissional para que possa esclarecer dúvidas e reduzir medos do binômio acompanhante e criança (Lucena et al., 2020).

Dessa forma, questiona-se em que nível e como o acompanhante é afetado pelo período de hospitalização da criança. Utilizando a questão norteadora "Quais os impactos psicoemocionais apresentados nos acompanhantes da criança quanto ao período de hospitalização pediátrica?", o presente estudo tem como objetivo geral analisar os impactos psicoemocionais no acompanhante da criança durante o período de hospitalização pediátrica. E como objetivos específicos, identificar os impactos psicoemocionais desse acompanhante frente a hospitalização.

\section{Metodologia}

O presente estudo e desenvolvimento do artigo foram realizados por meio de revisão sistemática. A revisão sistemática (RS) é um método de pesquisa e avaliação de um conjunto de dados provenientes de diferentes estudos que seguem protocolos específicos de busca que se encaixam em critérios de elegibilidade pré-definidos, com o objetivo de responder uma questão específica. Trata-se de um tipo de investigação focada em questões bem definidas, que visa identificar, selecionar, avaliar e sintetizar as evidências relevantes disponíveis. (De-La-Torre-Ugarte-Guanilo \& Takahashi, 2011; Galvão \& Ricarte, 2021).

Há alguns critérios para a aplicabilidade do método: ser exaustivo, contendo toda a literatura relevante na área; ser seguida uma metodologia rigorosa; ter pelo menos duas pessoas envolvidas para a análise de artigos e de dados, quanto sua qualidade metodológica e encaixe na seleção de trabalhos escolhidos de acordo com os critérios estabelecidos (Ercole, Melo \& Alcoforado, 2021; Donato \& Donato, 2019).

Na primeira etapa foi elaborada a questão norteadora através da estratégia do acrômico PICO (P=patient, I=intervention, $\mathrm{C}=$ control ou comparison $\mathrm{O}=$ outcome), neste estudo definido como: $\mathrm{P}=$ crianças, $\mathrm{I}=$ ansiedade na hospitalização, $\mathrm{Co}=$ pediatria.

A seleção e busca de artigos ocorreu por 3 juízes que realizaram a busca de forma independente. A identificação dos termos nos vocabulários controlados pelos Descritores em Ciências da Saúde (DECS) e Medical Subject Healding (MESH) na busca preliminar. Estes foram organizados com os operadores booleanos OR - permite o agrupamento/soma dos sinônimos e AND - faz a interseção dos termos. O operador NOT não foi utilizado neste estudo. O levantamento dos artigos nas mencionadas bases de dados ocorreu no mês de junho de 2021.

Os artigos que compõem o resultado deste estudo foram selecionados nas bases de dados: Banco de Dados em Enfermagem-Bibliografia Brasileira (BDENF - Enfermagem), Sistema Online de Busca e Análise de Literatura Médica (MEDLINE), Literatura Latino-Americana e do Caribe em Ciências da Saúde (LILACS).

No Portal de Periódicos da Capes foram empregadas as bases de dados Cumulative Index to Nursing and Allied Health Literature (CINAHL), por meio da Comunidade acadêmica Federada (Café) e Index Psicologia - Periódicos técnico científicos; para isso foi seguida as diretrizes do Preferred Reporting Items for Systematic Reviews and Meta-Analyzes (PRISMA), utilizando os descritores "criança, percepção, família ou pais e enfermagem". Os critérios de inclusão basearam-se em selecionar os artigos que relacionavam-se com o objetivo de interesse, que estivesse sido publicado nos últimos 5 anos, por apresentarem dados atuais sobre o foco de estudo, ou seja, de 2016 a 2021 e fosse encontrado nas linguagens em inglês, português ou espanhol. Os critérios de exclusão foram delineados em textos que não eram artigos (capítulos de livros, teses de doutorado, dissertações de mestrado, monografias, artigos de revisões integrativas e editoriais). 
A seleção da elegibilidade ocorreu por meio de uma triagem de artigos, com leitura de título e resumo, e uma etapa de confirmação por meio da leitura do manuscrito em forma de texto completo. Foi realizada a avaliação crítica da metodologia dos artigos para a revisão, seguida da síntese dos dados e participação dos juízes. A redação e apresentação dos dados ocorreu por meio de síntese descritiva considerando a análise dos artigos selecionados.

\section{Resultados e Discussão}

A partir da busca sistemática realizada, foram encontrados 15.372 artigos no total, previamente a aplicação dos critérios de inclusão e exclusão, sendo 73 no BDENF - Enfermagem, 106 no MEDLINE, 71 no LILACS, 15.120 no CINAHL e 2 no Index Psicologia. Aplicando o filtro para que mostrasse apenas artigos com texto completo disponível, 15.062 artigos foram excluídos e, após isso, adicionando os filtros de linguagem e filtro temporal, mais 165 foram retirados. Por fim, dos 145 artigos restantes, selecionamos aqueles que tinham relação com o objetivo de interesse, totalizando 6 artigos, e os outros 139 artigos foram excluídos. Dos 6 artigos selecionados 2 deles puderam ser encontrados na base de dados do BDENF - Enfermagem, 2 no LILACS e 2 no CINAHL, ficando o Index Psicologia e o MEDLINE sem apresentar artigos que se encaixasse nos critérios de inclusão utilizados. Portanto, encontra-se no fluxograma a seguir (Figura 1) a representação das fases da revisão sistemática.

Figura 1 - Fluxograma PRISMA com as fases da revisão sistemática.

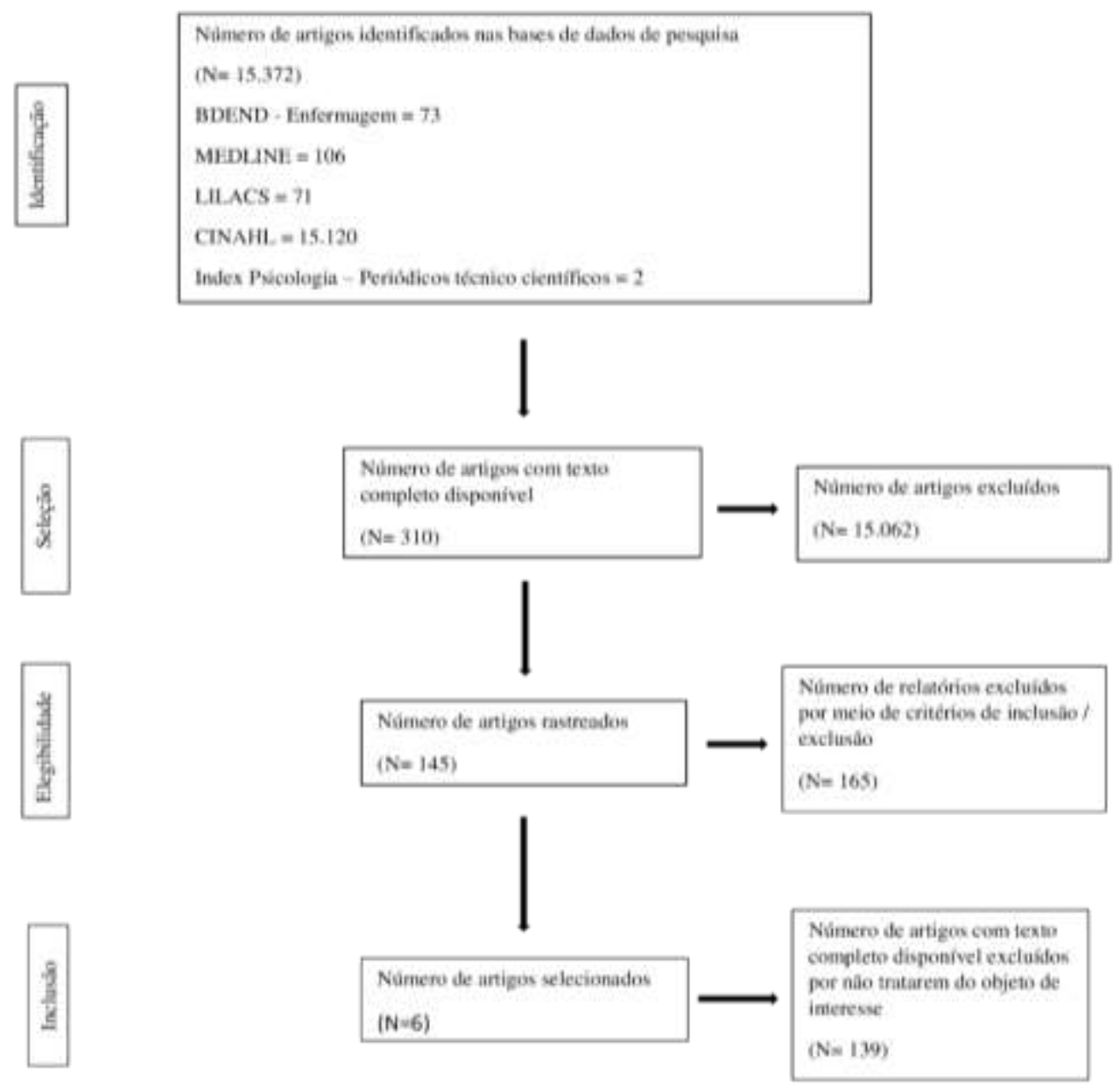

Fonte: Autores (2021).

O fluxograma apresenta as fases realizadas para construção da base de referências a serem utilizadas na revisão sistemática. Dessa forma, na fase de identificação houve a pesquisa nas bases de dados citadas na figura apenas com as palavras 
chaves determinadas; então, na seleção os artigos encontrados são filtrados de forma que fiquem apenas aqueles que tenham texto completo disponível; na fase de elegibilidade são aplicados os critérios de inclusão e exclusão; e por fim, na inclusão são selecionados os manuscritos que possuem relevância para a temática a ser abordada.

Ademais, representadas pelo quadro de referências abaixo (Quadro 1), há a descrição das importantes informações as quais retratam as referências selecionadas durante a pesquisa.

Quadro 1. Artigos selecionados na pesquisa.

\begin{tabular}{|c|c|c|c|c|c|}
\hline Título do artigo & Autor / Ano / País & Tipo de estudo & $\begin{array}{l}\text { Revista / Base } \\
\text { de dados }\end{array}$ & Objetivo & Resultados \\
\hline $\begin{array}{c}\text { Ansiedade de } \\
\text { crianças em } \\
\text { situação cirúrgica } \\
\text { e percepções } \\
\text { emocionais } \\
\text { reportadas por } \\
\text { seus } \\
\text { acompanhantes no } \\
\text { pré-operatório: um } \\
\text { estudo } \\
\text { exploratório }\end{array}$ & $\begin{array}{c}\text { Franzoi, M. A. H.; } \\
\text { Martins, G. } \\
2016 \\
\text { Brasil }\end{array}$ & $\begin{array}{c}\text { Estudo de } \\
\text { prevalência }\end{array}$ & $\begin{array}{l}\text { REME rev. } \\
\text { min. enferm / } \\
\text { BDENF - } \\
\text { Enfermagem; } \\
\text { LILACS }\end{array}$ & $\begin{array}{l}\text { Avaliar o estado de } \\
\text { ansiedade ré- } \\
\text { operatória de crianças } \\
\text { submetidas a cirurgias } \\
\text { eletivas e descrever as } \\
\text { percepções emocionais } \\
\text { de seus acompanhantes } \\
\text { relacionadas ao } \\
\text { processo cirúrgico. }\end{array}$ & $\begin{array}{l}\text { Verificou-se que mais de } 60 \% \text { das crianças estavam ansiosas. } \\
\text { A percepção emocional dos acompanhantes esteve associada } \\
\text { estatisticamente ao grau de parentesco com a criança, sendo } \\
\text { que mães e pais reportaram mais tensão relacionada } \\
\text { principalmente ao medo da anestesia e do procedimento } \\
\text { cirúrgico, decorrentes da falta de informações e orientações } \\
\text { sobre esses tópicos pela equipe de saúde. }\end{array}$ \\
\hline $\begin{array}{l}\text { Inserção do lúdico } \\
\text { como facilitador } \\
\text { da hospitalização } \\
\text { na infância: } \\
\text { percepção dos pais }\end{array}$ & $\begin{array}{l}\text { Rockembach, J.; } \\
\text { Espinosa, T.; } \\
\text { Cecagno, D.; Thumé, } \\
\text { E.; Soares, D. C. } \\
2017 \\
\text { Brasil }\end{array}$ & $\begin{array}{c}\text { Estudo } \\
\text { qualitativo, } \\
\text { descritivo e } \\
\text { exploratório }\end{array}$ & $\begin{array}{l}\text { J. nurs. health / } \\
\text { BDENF - } \\
\text { Enfermagem }\end{array}$ & 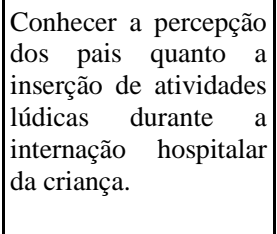 & $\begin{array}{l}\text { Da análise surgiram dois eixos temáticos as dificuldades } \\
\text { encontradas pelas crianças durante o período de internação } \\
\text { hospitalar, e, os benefícios da inserção de atividades lúdicas } \\
\text { durante a hospitalização. Considerações finais com a } \\
\text { utilização de atividades lúdicas, conclui-se que essas são } \\
\text { capazes de tornar o ambiente hospitalar menos ameaçador, } \\
\text { possibilitando resgatar sorrisos e alegria de ser criança. }\end{array}$ \\
\hline $\begin{array}{l}\text { Percepção dos } \\
\text { acompanhantes } \\
\text { das crianças } \\
\text { hospitalizadas } \\
\text { acerca do } \\
\text { brinquedo } \\
\text { terapêutico }\end{array}$ & $\begin{array}{l}\text { Silva, S. R. M.; } \\
\text { Santos, M. C. S.; } \\
\text { Silva, A. M.; Ferreira, } \\
\text { F. A.; Freitas, R. S. C.; } \\
\text { Gouveia, M. T.; } \\
\text { Rodrigues, W. F. G.; } \\
\text { Santos, R. E. A. } \\
\text { 2018 } \\
\text { Brasil }\end{array}$ & $\begin{array}{c}\text { Estudo } \\
\text { qualitativo, de } \\
\text { campo, } \\
\text { descritivo e } \\
\text { exploratório }\end{array}$ & $\begin{array}{l}\text { Rev. enferm. } \\
\text { UFPE on line / } \\
\text { CINAHL; } \\
\text { BDENF - } \\
\text { Enfermagem }\end{array}$ & 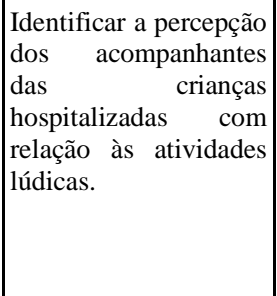 & $\begin{array}{l}\text { Elegeram-se, a partir da leitura e releitura das entrevistas, } \\
\text { quatro categorias }<<\text { A brinquedoteca como auxílio no } \\
\text { tratamento pediátrico }>>,<<\text { Utilização do Brinquedo } \\
\text { Terapêutico na realização dos procedimentos }>>;<< \\
\text { brinquedo terapêutico como suporte da assistência de } \\
\text { Enfermagem } \gg>,\langle<\text { O brinquedo e a aproximação entre o } \\
\text { profissional e a criança } \gg>\text {. }\end{array}$ \\
\hline $\begin{array}{l}\text { Percepção do } \\
\text { acompanhante do } \\
\text { paciente } \\
\text { pediátrico durante } \\
\text { a indução e o } \\
\text { despertar da } \\
\text { anestesia }\end{array}$ & $\begin{array}{c}\text { Binkowski, S.; de } \\
\text { Carvalho, G. P.; } \\
\text { Caregnato, R. C. A. } \\
2018 \\
\text { Brasil }\end{array}$ & $\begin{array}{c}\text { Estudo } \\
\text { exploratório, } \\
\text { descritivo, } \\
\text { qualitativo }\end{array}$ & $\begin{array}{c}\text { Rev. SOBECC / } \\
\text { BDENF - } \\
\text { Enfermagem; } \\
\text { LILACS }\end{array}$ & $\begin{array}{lrr}\text { Conhecer } & \text { as } \\
\text { percepções } & \text { de } \\
\text { acompanhantes } & \text { das } \\
\text { crianças } & \text { na } & \text { indução } \\
\text { anestésica } & \text { e } & \text { no } \\
\text { despertar da anestesia. }\end{array}$ & $\begin{array}{l}\text { Da análise de conteúdo dos diários de campo emergiram } \\
\text { duas categorias estratégias utilizadas pelos acompanhantes e } \\
\text { tranquilidade. As estratégias usadas foram choro, amor e } \\
\text { carinho. Das entrevistas emergiram duas categorias: } \\
\text { experiência positiva e experiência negativa, sendo a primeira } \\
\text { a mais destacada. }\end{array}$ \\
\hline $\begin{array}{c}\text { A utilização do } \\
\text { lúdico no cenário } \\
\text { da hospitalização } \\
\text { pediátrica }\end{array}$ & $\begin{array}{l}\text { Silva, M. K. C. O.; } \\
\text { Ferraz, L. C. C.; } \\
\text { Farias, M. B.; } \\
\text { Januario, J. K. C.; } \\
\text { Vieira, A. C. S.; } \\
\text { Moreira, R. T. F.; } \\
\text { Lucio, I. M. L. } \\
\text { 2019 } \\
\text { Brasil }\end{array}$ & $\begin{array}{c}\text { Estudo } \\
\text { qualitativo, } \\
\text { descritivo e } \\
\text { exploratório }\end{array}$ & $\begin{array}{l}\text { Rev. enferm. } \\
\text { UFPE on line / } \\
\text { CINAHL; } \\
\text { BDENF - } \\
\text { Enfermagem }\end{array}$ & $\begin{array}{l}\text { Descrever a percepção } \\
\text { da raipe } \\
\text { multiprofissional sobre } \\
\text { a utilização do lúdico e } \\
\text { dos fatores } \\
\text { interferem que na sua } \\
\text { prática no contexto do } \\
\text { cuidado à criança } \\
\text { hospitalizada. }\end{array}$ & $\begin{array}{l}\text { Revela-se que os profissionais percebem como o lúdico } \\
\text { ajuda a modificar o ambiente hospitalar, os sentimentos e o } \\
\text { processo de comunicação com a criança, influencia na } \\
\text { adesão às terapêuticas, a socialização, a promoção de } \\
\text { vínculos e a colaboração com a equipe, ainda que nem todos } \\
\text { se sintam preparados para incorporá-lo à sua rotina de } \\
\text { trabalho. }\end{array}$ \\
\hline $\begin{array}{c}\text { Perceção das mães } \\
\text { quanto às } \\
\text { necessidades e } \\
\text { cuidados } \\
\text { essenciais aos } \\
\text { filhos internados } \\
\text { num serviço de } \\
\text { Cuidados } \\
\text { Intensivos } \\
\text { Pediátricos }\end{array}$ & $\begin{array}{c}\text { Portugal, L. M. } \\
2020 \\
\text { Portugal }\end{array}$ & $\begin{array}{c}\text { Estudo } \\
\text { prognóstico / } \\
\text { Pesquisa } \\
\text { qualitativa }\end{array}$ & $\begin{array}{c}\text { BDENF - } \\
\text { Enfermagem }\end{array}$ & $\begin{array}{lr}\text { Compreender } & \text { a } \\
\text { natureza } & \text { das } \\
\text { experiências das mães } & \\
\text { e o modo como elas se } \\
\text { relacionam com } & \text { as } \\
\text { práticas diárias } & \text { de } \\
\text { cuidados à criança } & \text { e } \\
\text { família. } & \end{array}$ & $\begin{array}{l}\text { Evidenciou-se que as mães vivenciam o internamento do } \\
\text { filho com muito stress, ansiedade e angústia. As mães } \\
\text { esperançosas pela resolução do problema da criança, vendo } \\
\text { que os filhos estão estáveis, que são bem cuidados, mesmo } \\
\text { na sua ausência, sentem-se tranquilas. As mães querem } \\
\text { acompanhar os filhos e colaborar nos cuidados de forma } \\
\text { gradual, ganhando autonomia, sendo informadas de toda a } \\
\text { situação clínica da criança. Apesar de algumas } \\
\text { condicionantes do serviço, considerado de excelência, as } \\
\text { mães referem atenção por parte da equipa, quer para si quer } \\
\text { para os filhos, valorizando as competências técnicas e } \\
\text { humanas dos profissionais. }\end{array}$ \\
\hline
\end{tabular}

Fonte: Autores (2021). 
O quadro de referências apresenta todos os 6 artigos selecionados na revisão sistemática realizada, destacando o título dos artigos; os autores, ano e país de publicação; tipo de estudo; bases de dados e revista onde foi publicado; objetivos de cada artigo e seus respectivos resultados. Com as informações obtidas através do estudo e análise de cada artigo encontrado, foi realizada análise dos dados por meio de síntese descritiva considerando a análise dos artigos selecionados.

Estudos evidenciam que acompanhantes com grau de parentesco como mãe/pai, apresentaram um nível de tensão maior que os acompanhantes com outros graus de parentesco, como avós e tias, devido ao alto nível de vínculo com a criança, podendo aumentar o nível de nervosismo da criança no ambiente hospitalar, enquanto avós e tias podem ser mediadoras da tensão do local (Franzoi \& Martins, 2016). A ansiedade se faz presente principalmente devido a mudança repentina de rotina e ambiente, onde eles se vêem em uma situação atípica em um local que na maioria das vezes é desconhecido. A falta de privacidade é um incômodo e da mesma forma a mudança de hábitos e rotinas, incluindo também a substituições de algumas funções que eram realizadas em ambiente familiar pelos pais e no atual momento, devido a rotina hospitalar, são realizados pelos enfermeiros, fazendo com que os pais possam se sintam impotentes e tensos (Pedroso, 2017).

A mudança de hábitos e rotinas com a hospitalização, acaba gerando um estado de estresse e ansiedade que se acumula com o sofrimento oriundo da situação de internação e/ou cirurgia da criança, necessitando a adaptação em lidar com o risco e o medo de que possa acontecer intercorrências durante o período de internação. Gerando variadas manifestações emocionais, a hospitalização altera significativamente a relação entre os membros de uma família e somado a doença, podem gerar crises entre eles (Neves et al., 2018).

No momento da hospitalização a criança pode demonstrar diversos comportamentos, como a ansiedade, que não eram observados no contexto familiar. Como o medo da criança em separar-se dos pais podem resultar em alterações autonômicas, levando a desenvolvimento de sintomas como: tremores, alterações na frequência cardíaca e respiratórias, além dos distúrbios no sono e distúrbios alimentares (Moura et al., 2016). Com isso, pode ser ativado o eixo hipotálamo-hipofisário-adrenal, que determina o aumento dos níveis de glicocorticóides, podendo resultar na síndrome de cushing, devido ao excesso de produção do hormônio adrenocorticotrófico (ACTH). Os sinais e sintomas típicos incluem face em lua e obesidade do tronco, hematoma fácil e pernas e braços delgados e essa ativação pode contribuir para alterações no sistema imunológico (Moura et al., 2016).

A liberação excessiva ou escassa de neurotransmissores como serotonina, norepinefrina e GABA (ácido gama aminobutírico), podem gerar os sintomas de medo e ansiedade que variam entre tontura, sudorese, sensação de asfixia, tremores, palpitações, nervosismo e angústia (Bohneberger et al., 2016). Quanto maior o nível de estresse de um paciente, maior o período necessário para o desenvolvimento do processo de recuperação. Além disso, elevados níveis de ansiedade do paciente contribuem para o cancelamento de muitas cirurgias (Gonçalves, Sampaio \& Júnior, 2016).

Desta forma, é fundamental a utilização de estratégias que muita das vezes são realizadas pela equipe de enfermagem para minimizar a elevação dos anseios, como: ouvir a criança, e trabalhar mais a relevância do ouvir, com uma atenção ideal para ela, pois permite que ela acalme-se e expresse seus sentimentos, assim possibilitando melhor evolução e recuperação (Lima et al., 2020).

Assim, é notório que diversos sentimentos surgem antes da internação e a criança tende a compreender como uma ameaça. Além de realizar uma escuta ativa, existem estratégias capazes de desenvolver uma melhor experiência na hospitalização, como a realização de atividades na brinquedoteca; a prestação de assistência integral à saúde da criança, incluindo os acompanhantes na tomada de decisão; e a abordagem que engloba tanto os aspectos biológicos como os espirituais (Moura et al., 2016; Lima et al., 2020).

Visto isso, é notório que o impacto psicoemocional está presente tanto nas crianças quanto nos acompanhantes. A tensão dos acompanhantes se relaciona ao medo da internação em si, dúvidas sobre as orientações, sobre o procedimento o e a recuperação (Franzoi \& Martins, 2016). 
Dessa forma, fica evidente a relevância e a importância da equipe de enfermagem em desenvolver técnicas de assistência e cuidado, não só para a criança quanto também para seu acompanhante, a fim de diminuir ao máximo situações que são geradoras de conflitos e estresse, bem como implementar e disponibilizar ações e instrumentos que visem diminuir o estresse, a tensão, aumentar o conforto e sentimentos de segurança e confiança das crianças e acompanhantes, favorecendo assim uma melhor recuperação, minimizando possíveis complicações cirúrgicas.

A participação ativa e integral dos pais torna-se difícil devido a internação e pode, muitas das vezes, ocasionar sentimentos negativos e situações de estresse e ansiedade nos genitores (Binkowski et al., 2018).

As orientações e demais assuntos sobre o cuidado à criança são essenciais para que a família/acompanhantes sintam-se mais seguros. Para facilitar o enfrentamento da situação e para aumentar a sua confiança, ocorre a necessidade tanto da criança, quanto do acompanhante entender o que está ocorrendo, sendo de extrema importância que os profissionais envolvidos se atentem a isso e o acompanhante também auxilie no processo de passagem de informação para a criança, com uma linguagem adequada, devido ao seu vínculo e entendimento dos comportamentos já conhecidos pelo convívio. Essa prática de orientações é essencial para que tenha uma interação entre criança, o familiar e a equipe de enfermagem, facilitando a adaptação e a familiarização com o hospitalar em geral (Binkowski et al., 2018).

Contudo, a preparação psicológica para o procedimento cirúrgico é essencial para a criança e seus acompanhantes, pois possibilita um controle do desconhecido no momento de vulnerabilidade, quando o risco para saúde se faz presente. Os acompanhantes, especialmente as mães, querem ficar cientes e presentes, acompanhando e prestando os cuidados aos filhos, podendo ter uma maior autonomia após a alta, e esperam que sejam orientadas de toda a situação hospitalar e dos processos de internação, pré-operatório, trans e pós-operatório quando for o caso (Broering \& Crepaldi, 2018).

As mães relatam que a forma de falar com as crianças é primordial, informar a criança sobre seu quadro e com as devidas orientações, faz com que aceitem melhor a situação e os cuidados prestados (Portugal, 2019). Assim, como o acompanhante é orientado a relatar qualquer problema ou processo diferente com o paciente, é essencial que os profissionais de saúde oriente-os sobre o quadro do paciente em questão, podendo auxiliar no controle da ansiedade e preocupações futuras (Broering \& Crepaldi, 2018).

Os enfermeiros realizam ações para humanizar o atendimento do paciente suprindo suas necessidades biopsicossociais, juntamente com as necessidades do seu familiar, aperfeiçoando a relação entre a equipe, paciente e familiar, reconhecendo seus medos e suprindo suas dúvidas a respeito do tratamento proposto (SOBECC, 2017).

O período de hospitalização pediátrica é um momento potencialmente traumático, mesmo quando o acompanhante e a criança são orientados. Dessa forma, a enfermagem traça estratégias para a redução de riscos, entre elas pode-se destacar a contribuição da presença do acompanhante para a criança, um forte minimizador de ansiedade. Contudo, ainda há o impacto emocional em ambas as partes. Assim, o acompanhante e a equipe de enfermagem adotam estratégias para o enfrentamento desse momento e com isso, surge o destaque para essa especificidade de percepção do mesmo (Binkowski et al., 2018).

Desse modo, a estratégia adotada mais frequentemente vista foi o choro e a demonstração de afeto. Ademais, o acompanhamento de todo o período perioperatório em casos cirúrgicos também foi relatado como potencial estratégia facilitadora do enfrentamento do momento. A tranquilidade da criança também é relatada como um ponto positivo para o acompanhante (Binkowski et al., 2018).

A adoção de uma estratégia de enfrentamento parte da percepção do acompanhante de uma situação intimidadora, que esgote as suas opções de reação, bem como sobrecarregue seu estado emocional e físico; e que proporcione retomar o controle da crise. Ademais, vivenciar o período de hospitalização junto da criança não é um momento programado ou esperado, portanto as respostas de enfrentamento são aquelas para situações de crise acidentais (Kohlsdorf \& Junior, 2008).

Para mais, a falta de informação associada ao medo do desconhecido resultam no aumento da ansiedade, podendo gerar 
na criança alguma reação negativa, o que leva o acompanhante a adotar as estratégias focadas nos seus sentimentos, bem como das crianças, a exemplo a demonstração de afeto (Franzoi \& Martins, 2016; Sampaio, Marins \& Araujo, 2017).

Assim, a assistência de enfermagem durante a hospitalização deve abarcar todos os elementos relacionados à criança e ao acompanhante. Uma vez que ao dissociar o binómio criança-acompanhante, podem haver consequências negativas para a recuperação da criança, ao passo que é necessário que os serviços prestados sejam humanizados ao olhar o paciente como um todo (Neves et al., 2016).

Desse modo, a visita pautada na orientação quanto à rotina hospitalar e aos procedimentos que serão realizados (clínicos e cirúrgicos), tanto do acompanhante, quanto da criança deve ser realizada com vistas a diminuir a ansiedade de ambos e oferecer subsídios ao acompanhante para adotar estratégias de enfrentamento efetivas para si e para o paciente. Como também, é necessário, que todo o plano de cuidados da criança seja elaborado envolvendo o acompanhante (Sampaio et al., 2017).

Portanto, é necessário que o enfermeiro seja qualificado, enquanto profissional de pediatria, a fim de ser capaz de prestar cuidados em que a família seja o foco, visando a redução dos sentimentos negativos gerados pela hospitalização; bem como, de ser capaz de orientar e assistir essa família de modo que os próprios componentes desta rede elaborem e busquem suas próprias estratégias para o enfrentamento daquele momento. Vale ressaltar também, que o enfermeiro, deve e pode lançar mão do fomento à atividades terapêuticas reconhecidas, como leitura, atividades de cunho artístico, bem como a utilização de atividade lúdicas como o brinquedo terapêutico o que poderá favorecer a integração da equipe de enfermagem (Amatuzzi et al., 2019; Silva et al., 2019; Silva et al., 2018).

A presença dos acompanhantes e a realização de técnicas lúdicas auxiliam na diminuição da ansiedade das crianças no período de internação. Ao ter contato com os filhos e prestando os devidos cuidados, as mães desenvolvem sentimentos de bem estar e equilíbrio. Em contrapartida, neste momento é comum que as mães se encontrem em uma situação de insegurança e sofrimento, tornando-se frágeis e necessitando de atenção e apoio para se sentirem confiantes frente ao tratamento e à hospitalização de seus filhos. Além disso, as mães necessitam ser assistidas mediante informações perante suas preocupações e dúvidas devido a atual situação da criança, uma vez que o não acompanhamento do filho pode resultar em um aumento do estado de ansiedade nos pais. Dificulta-se assim o descanso e o tempo pessoal para que as mães fiquem em equilíbrio, para acalmaremse, participarem melhor e de forma integrada ao processo de cuidado e recuperação do filho, aumentando a confiança e esperança nos profissionais para boa recuperação (Portugal, 2019; Rockembach, 2017).

\section{Conclusão}

Dado o exposto, o período de hospitalização pediátrica é um movimento de ruptura na dinâmica cotidiana dos pacientes e seus acompanhantes. A hospitalização representa em muitos casos a quebra na rotina, nos espaços, nas relações sociais e interpessoais, e toda essa mudança pode gerar estresse, medo e outros impactos psicológicos.

A partir de um estudo de revisão sistemática conseguimos perceber que esse processo de hospitalização traz impactos psicoemocionais tanto no paciente pediátrico, como em seus acompanhantes, gerando ansiedade, distúrbios no sono, na alimentação entre outras complicações que podem interferir diretamente no seu tratamento. Os familiares sofrem bastante com esse processo, e são acometidos por incertezas, inseguranças, sentimento de impotência, entre outros, e tudo isso pode refletir diretamente na criança e no seu processo de internação. Para controlar tais sentimentos e fazer da hospitalização um processo menos traumático, os estudos apontam que a enfermagem deve atuar com variadas ações de cuidado, sendo um dos maiores aliados o fornecimento de informação a fim de orientar e acalmar os acompanhantes. Tais profissionais devem estar aptos para acolher a criança e seu acompanhante, e conceder assistência de acordo com o que cada família precisa, desenvolvendo artifícios que possibilitem minimizar possíveis dificuldades e intercorrências. 
Desse modo, a enfermagem deve passar por um atendimento mais humanizado, construindo e tecendo estratégias que visam diminuir os efeitos psicoemocionais que a hospitalização produz nos pacientes e seus acompanhantes, posto que a atenção e o cuidado desses profissionais numa abordagem humanizada podem atenuar o sofrimento gerado por esse período.

Este estudo contribui para pesquisar os impactos psicoemocionais do acompanhante e da criança frente a cirurgia pediátrica e hospitalização, evidenciando a assistência da enfermagem que utiliza estratégias as quais contribuem para um maior equilíbrio emocional do acompanhante e por consequência da criança. Assim como, colaborar para o progresso de novos estudos nessa área.

Deve-se considerar algumas limitações do estudo que podem dificultar a sua generalização de resultados, como a diversidade da realidade populacional a qual implica nas possibilidades das estratégias adotadas.

Nesse cenário, faz presente a demanda de uma pesquisa que proporcione apresentar artifícios os quais possibilitem minimizar os problemas que possam surgir no ambiente hospitalar, devido a situação de estresse e ansiedade vivenciada no momento cirúrgico. Assim, os enfermeiros deverão pensar em estratégias de acolhimento a criança e seu acompanhante como forma de minimizar o medo, ansiedade, estresse e complicações, favorecendo tratamentos no período perioperatório.

\section{Referências}

Amatuzzi, E., Souza, M. A., \& Melo, L. L. (2019). Vivências de famílias de crianças em intraoperatório: a arte como possibilidade de cuidado. Rev enferm UERJ, 27(36678), 1- 8. https://doi.org/10.12957/reuerj.2019.36678

Binkowski, S., De Carvalho, G. P., \& Caregnato, R. C. A. (2018). Percepção do acompanhante do paciente pediátrico durante a indução e o despertar da anestesia. Revista SOBECC, 23(1), 14-20. Recuperador de https://revista.sobecc.org.br/sobecc/article/view/386/pdf

Bohneberger, G., Locatelli, D., Caré, L. L., Sales, J., Oliveira, T. G. de, \& Nardi, A. (2017). Alterações fisiológicas da ansiedade e do medo e implicações na odontologia. Ação Odonto, 2, 14. https://portalperiodicos.unoesc.edu.br/acaodonto/article/view/12489

Broering, C., \& Crepaldi, M. A. (2018). Percepções e informações das mães sobre a cirurgia de seus filhos. Fractal: Revista de Psicologia, 30(1), 3-11. https://doi.org/10.22409/1984-0292/v30i1/1434

Carvalho, C. A. L. B., Carvalho, A. A., Nogueira, P. L. B. \& Aguilar-Nascimento, J. E. (2017). Mudando paradigmas em jejum pré-operatório: resultados de um mutirão em cirurgia pediátrica. ABCD. Arquivos Brasileiros de Cirurgia Digestiva, 30(1), 7-10. https://doi.org/10.1590/0102-6720201700010003

De-La-Torre-Ugarte-Guanilo, M. C., Takahashi, R. F. \& Bertolozzi, M. R. (2011). Revisão sistemática: noções gerais. Revista da Escola de Enfermagem da USP, 45(5), 1260-1266. https://doi.org/10.1590/S0080-62342011000500033

Donato, H., Donato, M. (2019). Etapas na Condução de uma Revisão Sistemática. Acta Médica Portuguesa, 32(3), 227-235. https://doi.org/10.20344/amp.11923

Ercole, F. F., Melo, L. S. de, \& Alcoforado, C. L. G. C. (2014). Revisão integrativa versus revisão sistemática. Reme: Revista Mineira de Enfermagem, 18(1). 12-14. https://doi.org/10.5935/1415-2762.20140001

Franzoi, M. A. H., \& Martins, G. (2016). Ansiedade de crianças em situação cirúrgica e percepções emocionais reportadas por seus acompanhantes no préoperatório: um estudo exploratório. Revista Mineira de Enfermagem, 20(984), 1-9. https://cdn.publisher.gn1.link/reme.org.br/pdf/e984.pdf

Galvão, M. C. B., \& Ricarte, I. L. M. (2019). Revisão sistemática da literatura: conceituação, produção e publicação. Logeion: Filosofia Da Informação, 6(1), 57-73. https://doi.org/10.21728/logeion.2019v6n1.p57-73

Gonçalves, K. K. N., Silva, J. I. d., Gomes, E. T., Pinheiro, L. L. d. S., Figueiredo, T. R., \& Bezerra, S. M. M. d. S. (2016). Ansiedade no período pré-operatório de cirurgia cardíaca. Revista Brasileira de Enfermagem, 69(2), 397-403. https://doi.org/10.1590/0034-7167.2016690225i

Gonçalves, R. A., Sampaio, C., \& Júnior, H. (2016). Determinação dos fatores da suspensão de cirurgia e suas contribuições para assistência de enfermagem. Revista de Pesquisa: Cuidado é Fundamental Online, 8(3), 4813-4820. https://doi.org/10.9789/2175-5361.2016.v8i3.4813-4820

Kohlsdorf, M. \& Junior, Á. L. C. (2008). Estratégias de enfrentamento de pais de crianças em tratamento de câncer. Estudos de Psicologia, 25(3), 417-429. https://doi.org/10.1590/S0103-166X2008000300010

Lucena, J. S., Silva, A. B. C. d., Marques, M. J., Gomes, B. d. M. R., Sousa, T. D. d. A., \& Pereira, E. B. F. e. (2020). Ansiedade na cirurgia vascular e ações de educação em saúde no pré operatório. Revista Enfermagem Digital Cuidado e Promoção da Saúde, 5(1), 374-80 http://dx.doi.org/10.1590/0034$7167.2016690225 \mathrm{i}$

Morero, J. A. P., Bragagnollo, G. R. \& Santos, M. T. S. (2018). Estratégias de enfrentamento: uma revisão sistemática sobre instrumentos de avaliação no contexto brasileiro. Rev Cuid, 9(2), 2257- 2268. https://doi.org/10.15649/cuidarte.v9i2.503

Moura, L. A., Dias, I. M. G., \& Pereira, L, V. (2016). Prevalência e fatores associados à ansiedade pré-operatória em crianças de 5 a 12 anos. Latino - Americana de Enfermagem, 24, 1-7. http://dx.doi.org/10.1590/1518-8345.0723.2708 
Research, Society and Development, v. 11, n. 3, e20411326259, 2022

(CC BY 4.0) | ISSN 2525-3409 | DOI: http://dx.doi.org/10.33448/rsd-v11i3.26259

Neves, F. G. \& Moraes J. R. M. M. \& Morais, R. C. M. M. \& Souza, T. V. \& Ciuffo, L. L. \& Oliveira, I. C. S. (2016). O trabalho da enfermagem em emergência pediátrica na perspectiva dos acompanhantes. Esc. Anna Nery Rev Enferm, 20(3). 1-7. https://doi.org/10.5935/1414-8145.20160063

Neves, L., Gondim, A. A., Soares, S. C. M. R., Coelho, D. P., \& Pinheiro, J. A. M. (2018). O impacto do processo de hospitalização para o acompanhante familiar do paciente crítico crônico internado em Unidade de Terapia Semi-Intensiva. Escola Anna Nery, 22(2), 1-8. https://doi.org/10.1590/2177-9465-EAN2017-0304

Pedroso, R. M. C. J. (2017). Impacto da parceria de cuidados para a criança hospitalizada e sua família. International Journal of Developmental and Educational Psychology. Revista INFAD de Psicologia., 3(1), 225-232. https://doi.org/10.17060/ijodaep.2017.n1.v3.991

Portugal, L. M. (2019). Perceção das mães quanto às necessidades e cuidados essenciais aos filhos internados num serviço de Cuidados Intensivos Pediátricos. (Dissertação de mestrado). Escola Superior de Enfermagem de Coimbra, Coimbra, Portugal.

Rothrock, J. C. (2021). Alexander Cuidados de enfermagem ao paciente cirúrgico. Elsevier.

Rockembach, J. A., Espinosa, T. A., Cecagno, D., Thumé, E., Soares, D. C. (2017). Inserção do lúdico como facilitador da hospitalização na infância: percepção dos pais. Journal of Nursing and Health. 7(2), 117-126. https://doi.org/10.15210/jonah.v7i2.7646.

Sampaio, C. E. P., Gomes, A. M. T., Souza, C. L. A. d., Mattos, M. F. C. d., \& Holanda, J. S. d. (2021). Vivendo com medo, preocupação e ansiedade: representações de cirurgia para familiares de crianças no pré-operatório. Research, Society and Development, 10 (11), 1-10. https://doi.org/10.33448/rsdv10i11.19671

Sampaio, C. E. P., Marins, T. G. de, \& Araujo, T. V. F. L. de (2017). Nível de ansiedade de crianças no pré-operatório: avaliação segundo a Escala de Yale modificada. Rev enferm UERJ, 25, 1-6. http://dx.doi.org/10.12957/reuerj.2017.28069

Silva, M. K. C. de O., Ferraz, L. C. C., Farias, M. B. de, Januario, J. K. C., Vieira, A. C. S., Moreira, R. T. de F., \& Lucio, I. M. L. (2019). A utilização do lúdico no cenário da hospitalização pediátrica. Rev. enferm. UFPE on line, 13(1), [1-7]. http://dx.doi.org/10.5205/1981-8963.2019.238585

Silva, S. R. de M., Santos, M. C. S. dos, Silva, A. M. da, Ferreira, F. A., Freitas, R. de S. C., Gouveia, M. T. \& Santos, R. E. A. dos. (2018). Percepção dos acompanhantes das crianças hospitalizadas acerca do brinquedo terapêutico. Rev. enferm. UFPE on line, 12(10). 2703-2709. https://doi.org/10.5205/1981-8963v12i10a235934p2535-2545-2018

SOBECC. (2017). Diretrizes de Práticas em Enfermagem Cirúrgica e Processamento de Produtos para a Saúde (7a ed.). SOBECC. 